\title{
Livoneca redmanni Leach (Isopoda, Cymothoidae) e Rocinela signata Schioedte \& Meinert (Isopoda, Aegidae), ectoparasitos de Scomberomorus brasiliensis Collette, Russo \& Zavala-Camin (Ostheichthyes, Scombridae) no Rio Grande do Norte, Brasil
}

\author{
José T. A. X. de Lima ${ }^{1,3}$, Sathyabama Chellappa ${ }^{1} \&$ Vernon E. Thatcher ${ }^{2}$ \\ 1 Programa de Pós-Graduação em Bioecologia Aquática, Departamento de Oceanografia e Limnologia, Centro de Biociências, \\ Universidade Federal do Rio Grande do Norte. Praia de Mãe Luiza, Via Costeira, 59014-100 Natal, Rio Grande do Norte, \\ Brasil.E-mail: ximenesvet@ig.com.br; bama@dol.ufrn.br \\ 2 Departamento de Zoologia, Universidade Federal do Paraná. Caixa Postal 19020, 81531-980 Curitiba, Paraná, Brasil. \\ E-mail: thatcher@ufpr.br \\ ${ }^{3}$ Autor correspondente.
}

\begin{abstract}
Livoneca redmanni Leach (Isopoda, Cymothoidae) and Rocinela signata Schioedte \& Meinert (Isopoda, Aegidae), ectoparasites of Scomberomorus brasiliensis Collette, Russo \& Zavala-Camin (Ostheichthyes, Scombridae) in Rio Grande do Norte, Brazil. Among the crustacean isopoda there exists ectoparasites which are easy to observe due to their size. They cause lesions on the host that could lead to death. The present study verified the occurrence of parasitic isopods on the fish Serra Spanish mackerel, Scomberomorus brasiliensis (Collette, Russo \& Zavala-Camin, 1978) from the coastal waters of Rio Grande do Norte, Brazil. Indices of parasitic infection by isopods were calculated and correlated to the body size, weight and the stage of gonadal maturity of the host. Livoneca redmanni (Leach, 1818) (Cymothoidae) and Rocinela signata (Schioedte \& Meinert, 1879) (Aegidae) were registered for the first time in the coastal waters of Rio Grande do Norte. L. redmanni was encountered both in the oral cavity and in the gill chambers of the host, whereas $R$. signata was found only in the gill chambers. Parasitic isopods were encountered on $31 \%$ of S. brasiliensis captured, wherein $86 \%$ was L. redmanni and $14 \%$ was $R$. signata. The maximum parasitic intensity was four with a minimum of one isopods per host. The isopods showed a preference for immature and maturing stages of the host $S$. brasiliensis.

KEY WORDS. Gill chamber, isopod marine, oral cavity, parasites, parasitic infection.
\end{abstract}

RESUMO. Entre Isopodas Crustaceas existem ectoparasitos que são de fácil observação devido ao seu tamanho. Eles causam lesões mecânicas no hospedeiro que podem resultar em morte. Este trabalho verificou a existência de parasitismo por isópodos no peixe serra, Scomberomorus brasiliensis (Collette, Russo \& Zavala-Camin, 1978) das águas costeiras do Rio Grande do Norte, Brasil. Os índices de infestação parasitária dos isópodos foram calculados e relacionados com o tamanho, peso e o estado gonadal do hospedeiro. Livoneca redmanni (Leach, 1818) (Cymothoidae) e Rocinela signata (Schioedte \& Meinert, 1879) (Aegidae) foram registradas pela primeira vez nas águas costeiras do Rio Grande do Norte. Os locais de fixação de $L$. redmanni no hospedeiro foram a cavidade bucal e a câmara branquial, enquanto $R$. signata sempre foi encontrada na câmara branquial. Dos exemplares de S. brasiliensis capturados $31 \%$ estavam parasitados, onde $86 \%$ eram de $L$. redmanni e $14 \%$ de $R$. signata. Foi registrado uma intensidade mínima de um e máxima de quatro isópodos por hospedeiro. Os isópodos apresentaram uma preferência pelos hospedeiros imaturos e em maturação de $S$. brasiliensis.

PALAVRAS CHAVE. Câmara branquial, cavidade bucal, isopodas marinhos, parasitos, infestação parasitária.

Na ictiofauna marinha da região sul do Brasil foi registrado a ocorrência de espécies de ectoparasitos de Cymothoidae (ThAtcher et al. 2003) e as espécies de Aegidae foram encontradas no sudeste do Brasil (MoreIra 1972, 1977). Os crustáceos isópodos parasitos habitam a câmara branquial, cavidade bucal e tegumento dos peixes, sendo facilmente detectados a olho nu (Eiras et al. 2000, Thatcher 2000). As formas jovens dos parasitos podem penetrar mais intensamente abaixo das escamas 
dos peixes, e à medida que crescem ocupam boa parte da cavidade visceral, o que compromete o crescimento do animal. A quantidade e o local de fixação dos parasitos, bem como o tamanho do hospedeiro, causa alterações na capacidade respiratória dos peixes, emagrecimento, redução na taxa de crescimento e alteração na capacidade natatória. Em geral os parasitos acarretam lesões, cujos resultados podem levar à instalação de infecções que propiciam a morte do peixe (PAVANelli et al. 1999).

Scomberomorus brasiliensis é uma espécie marinha de valor comercial que habita a maior parte do litoral brasileiro, com exceção das extremidades do norte e do sul (Zavala-CAmin 1983). Os dados da produção pesqueira demonstram que a quantidade desembarcada apresentou uma tendência de crescimento no Rio Grande do Norte, Ceará, Piauí, Maranhão e Pará (IBAma 2003).

Scomberomorus brasiliensis foi uma das espécies de peixes marinhos das águas costeiras do Rio de Janeiro, Brasil, que foram estudadas em relação à biodiversidade parasitária (LuQUE et al. 2004), no entanto, existe uma carência de informações sobre o parasitismo do Scombridae no Rio Grande do Norte.

Foi verificada a existência do parasitismo por isópodos em S. brasiliensis capturados nas águas costeiras do Rio Grande do Norte. Os índices de infestação parasitária dos isópodos foram calculados e relacionados com o tamanho, peso e o estado gonadal do serra.

\section{MATERIAL E MÉTODOS}

As coletas dos peixes-serra foram realizadas mensalmente durante o período de agosto de 2003 a julho de 2004, nas águas costeiras da Praia de Ponta Negra (0552'30"S e $\left.35^{\circ} 08^{\prime} 00^{\prime \prime} \mathrm{W}\right)$, localizada na região urbana no município de Natal, Rio Grande do Norte, Brasil. Os peixes foram capturados por meio de redes de arrasto de praia do tipo tresmalho com $110 \mathrm{~m}$ de comprimento, $3 \mathrm{~m}$ de altura com malha central de $10 \mathrm{~mm}$ e $70 \mathrm{~mm}$ nas extremidades. A rede foi lançada a $100 \mathrm{~m}$ de distância da praia, em uma profundidade de $5 \mathrm{~m}$, utilizando uma pequena balsa chamada catraia. Todo o processo, desde a arrumação até a retirada da rede com os peixes, dura em torno de uma hora e 30 minutos. Mensalmente as coletas foram realizadas com o uso de três arrastos-de-praia consecutivos com o esforço de seis a 12 pessoas.

No Laboratório de Ictiologia da Universidade Federal do Rio Grande do Norte os peixes foram medidos (comprimento total em $\mathrm{mm}$ ) e pesados (peso total em $\mathrm{g}$ ). Os valores morfométricos e merísticos dos peixes foram utilizados para confirmar a taxonômia da espécie (FonTeles-Filho 1988). Posteriormente, os peixes foram dissecados para a sexagem e a determinação dos estádios de maturação gonadal seguindo a metodologia de VAzzoler (1996).

Foi realizada uma procura minuciosa de ectoparasitos na superfície corporal, na cavidade bucal e na câmara branquial dos peixes, seguindo as técnicas propostas por Eiras et al. (2000). Os isópodos foram cuidadosamente retirados e acondicionados em tubos de ensaio com álcool a 70\%, posteriormente sen- do identificados através da utilização de chaves de identificação (Thatcher 2000).

Os cálculos dos índices parasitários foram efetuados a partir do formulário de necropsia dos peixes que contém informações tais como: o número de parasitos isópodos em cada hospedeiro e o respectivo local de fixação destes. Os índices calculados foram os seguintes: Prevalência $\left(P=n p p / n_{x} 100\right)$, onde $\mathrm{npp}$ = número de peixes parasitados, e $\mathrm{n}=$ número de peixes examinados; Intensidade (mínimo e máximo); Intensidade média de parasitos por peixes $(\mathrm{Im}=\mathrm{tpp} / \mathrm{npp})$, onde tpp = número total de parasitos na amostra; Abundância (A = tpp/ $\mathrm{ntp}$ ), onde $\mathrm{ntp}=$ número total de peixes (parasitados ou não) na amostra (Bush et al. 1997). Foram registradas também a relação parasito-hospedeiro, o tamanho, o peso e os estádios de maturação gonadal dos hospedeiros.

\section{RESULTADOS E DISCUSSÃO}

A variação do peso total de $S$. brasiliensis capturados neste estudo foi de 15 a 3,385 g e a de comprimento total foi de 135 a 805 mm, e observou-se a presença dos estádios gonadais imaturos, em maturação, maduros e esvaziados.

Os parasitos identificados em $S$. brasiliensis pertencem a duas famílias: Cymothoidae, com a espécie L. redmanni (Leach, 1818) e Aegidae, com a espécie R. signata (Schioedte \& Meinert, 1879).

Dos peixes serras capturados $31 \%$ estavam parasitados, totalizando 67 isópodos fixados nas lamelas branquiais do peixe; a maioria estava na câmara branquial (Figs 1-2) e apenas um na cavidade bucal do peixe (Fig. 3). Uma fêmea da serra apresentava duas espécies de isópodos aderidas às brânquias, o que corresponde a um caso de coexistência parasitária.

Foram constatadas algumas alterações na cavidade branquial, dentre estas: lesões mecânicas com produção excessiva de secreção mucosa e falha nos filamentos brânquias ou lacunas no arco branquial, em decorrência da fixação dos isópodos nestes locais (Fig. 4).

Em termos de ocorrência, foram constatados $86 \% \mathrm{~L}$. redmanni (Cymothoidae) (Fig. 5) e 14\% de R. signata (Aegidae) (Fig. 6) nos indivíduos de $S$. brasiliensis parasitados. Destes peixes foram observados 23 machos e 21 fêmeas, com uma proporção sexual equivalente a 1:1.

No presente estudo foi verificada a ausência de correlação entre o parasito e o sexo do hospedeiro, além de ser registrado a co-existência de espécies de parasitos em peixes marinhos igualmente constatados por OLIVA \& LUQUE (1998) e LUQUE et al. (2004).

\section{Índices de infestação parasitária}

Foi observada a maior prevalência de Cymothoidae em dezembro de 2003 , onde $75 \%$ dos peixes capturados estavam parasitados (Fig. 7). No mesmo mês as serras apresentaram a maior intensidade média de 2,3 L. redmanni por peixe parasitado (Fig. 8). A maior abundância de L. redmanni ocorreu em maio de 2004, com valor de 1,1 (Fig. 9). 

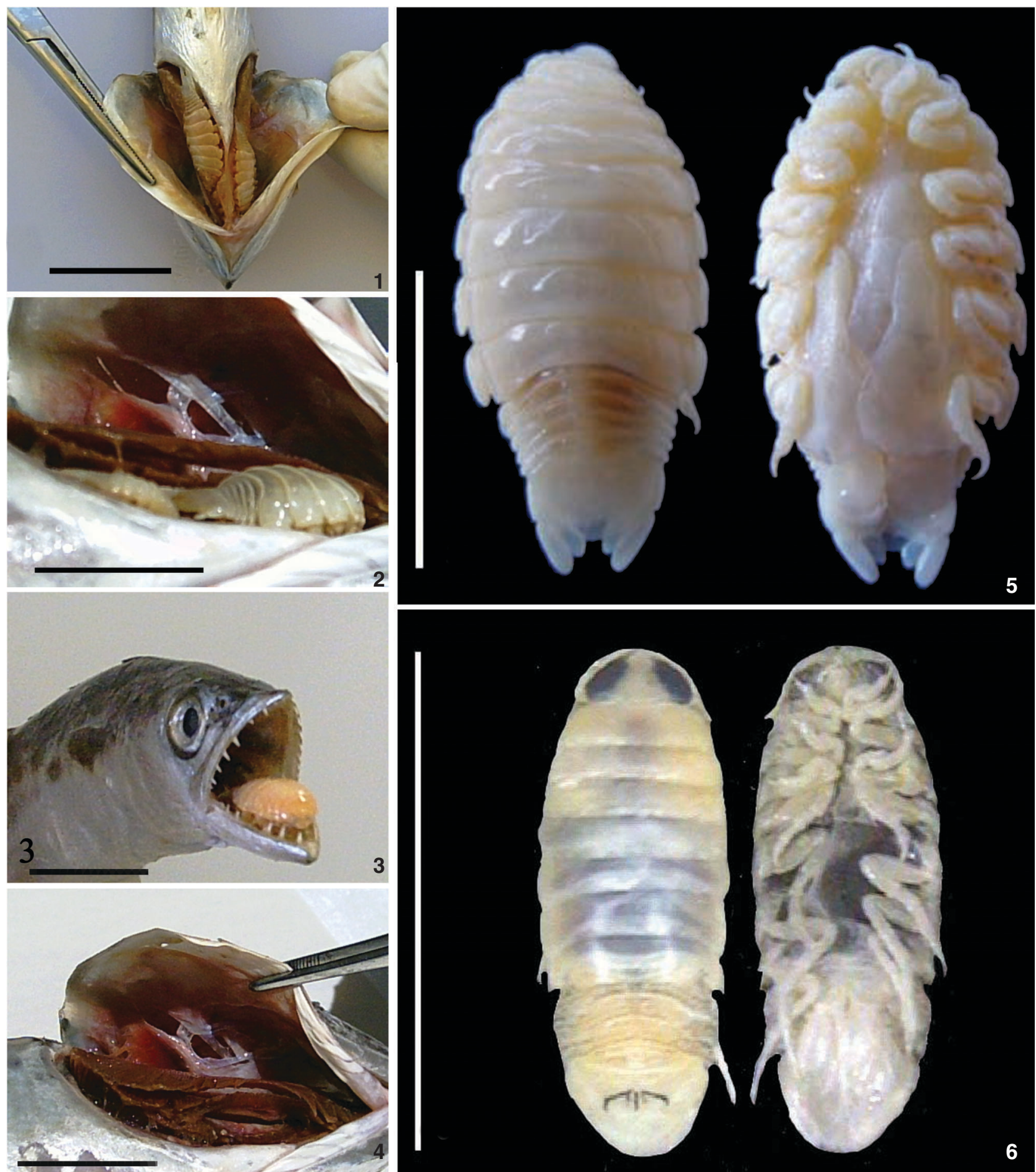

Figuras 1-6. Parasitos Livoneca redmanni e Rocinela signata em Scomberomorus brasiliensis capturados nas águas costeiras do Rio Grande do Norte. (1-2) Livoneca redmanni aderida às brânquias através da câmara branquial (escala = 20 mm); (3) Livoneca redmanni aderido às brânquias pela cavidade bucal (escala = $20 \mathrm{~mm}$ ); (4) lacunas nos arcos branquiais do hospedeiro, decorrente de lesões mecânicas pela fixação do isópodo (escala = $20 \mathrm{~mm}$ ); (5) posição dorsal e ventral de Livoneca redmanni (lado a lado) (escala = $10 \mathrm{~mm}$ ); (6) posição dorsal e ventral de Rocinela signata (lado a lado) (escala $=5 \mathrm{~mm}$ ).

Revista Brasileira de Zoologia 22 (4): 1104-1108, dezembro 2005 

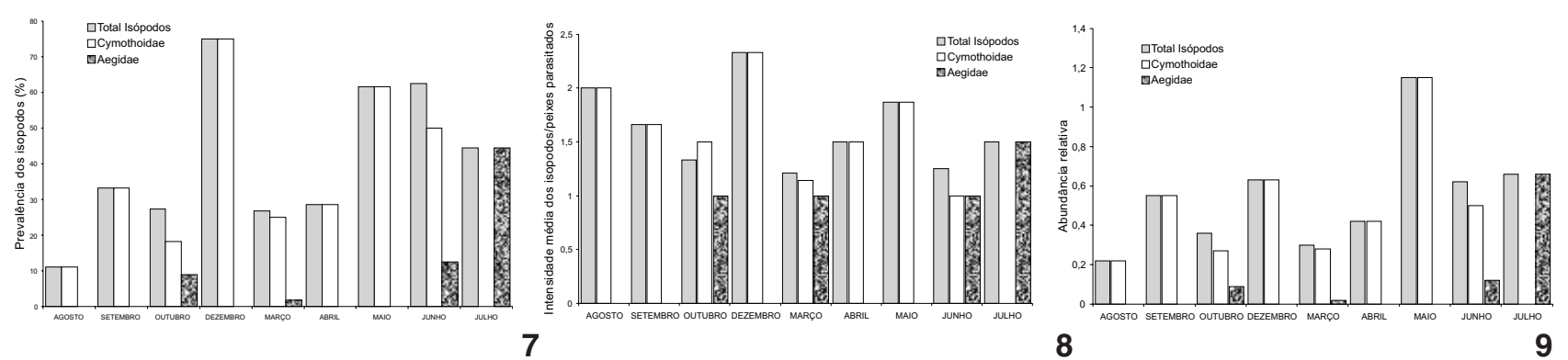

Figuras 7-9. Índices de infestação parasitária de Livoneca redmanni e Rocinela signata na serra, Scomberomorus brasiliensis capturados nas águas costeiras do Rio Grande do Norte, no período de agosto de 2003 a julho 2004: (7) variação da prevalência; (8) variação da intensidade média; (9) variação da abundância relativa.
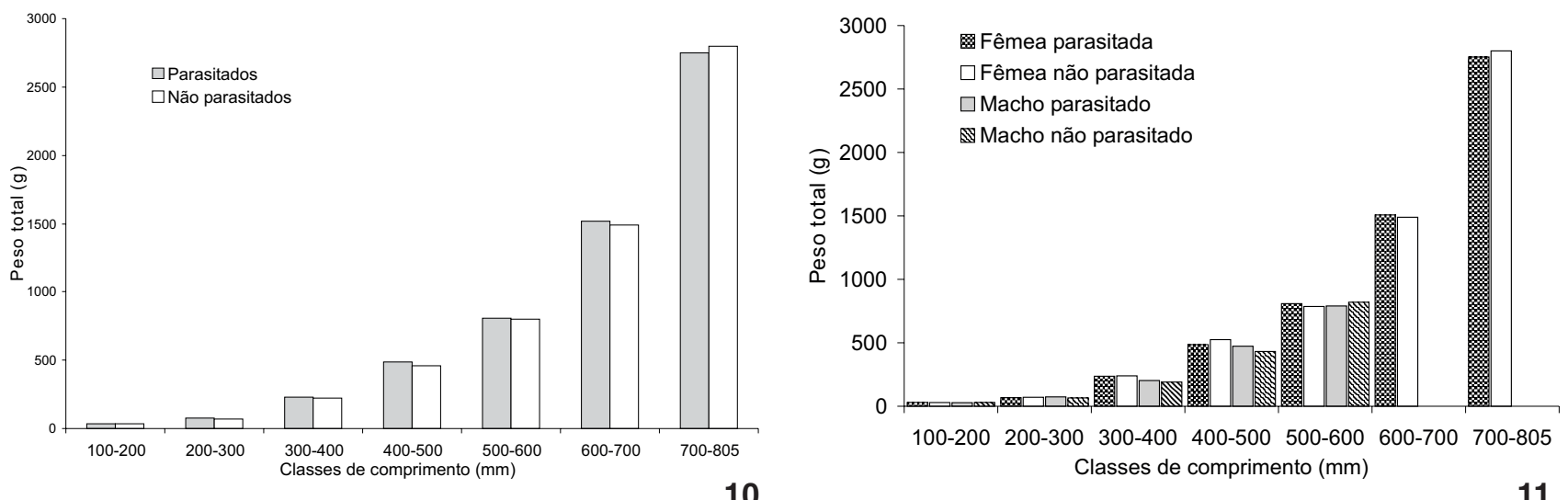

Figuras 10-11. Peso total (g) e classe de comprimento $(\mathrm{mm})$ de serras machos e fêmeas parasitados e não parasitados: (10) machos e fêmeas não parasitados e parasitados por isópodos nas águas costeiras do Rio Grande do Norte em relação às classes de comprimento total (mm); (10) serras não parasitados e parasitados por isópodos nas águas costeiras do Rio Grande do Norte em relação às classes de comprimento total $(\mathrm{mm})$.

A maior prevalência de Aegidae ocorreu em julho de 2004, onde $44,44 \%$ dos peixes capturados estavam parasitados (Fig. 7). No mesmo mês as serras tiveram a maior intensidade média de 1,5 R. signata por peixe parasitado (Fig. 8). A maior abundância de $R$. signata foi registrado em julho de 2004, equivalente a 0,7 (Fig. 9).

O número mínimo de isópodos por peixe foi de um e a máxima foi de quatro em um mesmo hospedeiro. Em geral os isópodos apresentaram uma intensidade média de 1,5 isópodos por peixe parasitado. Neste estudo foi registrado uma abundância geral de 0,5 parasito por peixe. Este estudo está em concordância com os resultados relatados por CAVALCANTI et al. (2003, 2004) na serra, S. brasiliensis e palombeta, Shloroscombrus chrysurus (Linnaeus, 1766).

\section{Relação parasitismo com tamanho, peso e estádio de desen- volvimento das gônadas do hospedeiro}

As classes de comprimentos totais dos peixes que mais estavam parasitados variaram entre 200 a $400 \mathrm{~mm}$, com um nú- mero máximo de quatro isópodos por hospedeiro com intensidade média em 2,15 isópodos por peixe parasitado. O local de fixação pode determinar o emagrecimento do indivíduo parasitado em ambiente confinado (PAvanelli et al. 1999). No entanto, neste estudo em ambiente natural, a fixação dos isópodos na cavidade bucal e na câmara branquial não influenciou o emagrecimento do hospedeiro, haja vista que não foram observadas diferenças significativas ao nível de 0,05 ( $p$ valor $>0,05$ ) com o teste-t entre comprimento total, peso total e sexo dos peixes parasitados por isópodos e os não parasitados (Figs 10 e 11).

Os isópodos apresentaram uma preferência pelos hospedeiros em estádio inicial de desenvolvimento gonadal (51\%), seguido pelos peixes em estádio em maturação (38\%), estádios maduros (9\%) e esvaziados (2\%) (Fig. 12). A preferência dos parasitos pelos hospedeiros mais jovens possivelmente ocorre por estes apresentarem mais recursos energéticos, em relação aos maduros e esvaziados. Estes últimos apresentam menos reservas energéticas devido à mobilização destas para a maturação gonadal e a reprodução (Chellappa et al. 1995, HunTingFord et al. 2001). 
Este estudo é o primeiro registro de isópodos marinhos das espécies $L$. redmanni e $R$. signata no estado do Rio Grande do Norte.

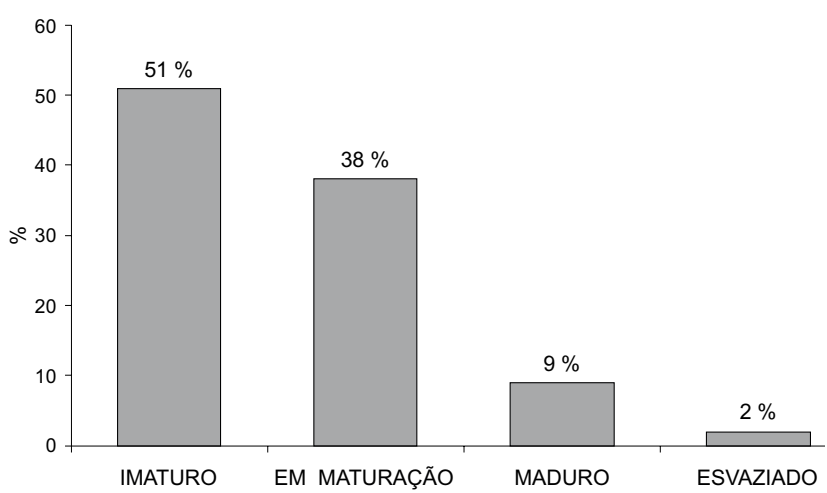

Figura 12. Número percentual dos peixes serra, Scomberomorus brasiliensis, parasitados por isópodos, em relação aos estádios de maturação gonadal.

\section{AGRADECIMENTOS}

O primeiro autor agradece a CAPES/MEC pela bolsa de Mestrado concedida, a Universidade Federal do Rio Grande do Norte e ao Departamento de Zoologia da Universidade Federal do Paraná, pelo apoio proporcionado. A segunda autora agradece ao Conselho Nacional de Desenvolvimento Científico e Tecnológico (CNPq) pela bolsa de Produtividade em Pesquisa.

\section{REFERÊNCIAS BIBLIOGRÁFICAS}

Bush, A.O.; K.D. Laffehty; J.M. Lotz \& A.W. Shostak. 1997. Parasitology meets ecology on its own terms: Margolis et al. Revisted. Journal of Parasitology, Washington, 83 (4): 575-583.

Cavalcanti, E.T.S.; J.T.A.X. Lima; S. Chellappa \& G.C. Pavanelli. 2003. Ectoparasitos nos peixes marinhos serra, Scomberomorus brasiliensis (Osteichthyes: Scombridae) e palombeta, Chloroscombrus chrysurus (Osteichthyes: Carangidae) do litoral de Ponta Negra, RN, p. 266-267. In: V. Claudino-SALEs; I. M. Tonini \& E. W.C. Dantas (Eds). Ecologia do Brasil. Fortaleza, Universidade Federal do Ceará, 646p.

Cavalcanti, E.T.S.; G.C. Pavanelli; S. Chellappa \& R.M. Takemoto. 2004. Comunidade de metazoários ectoparasitos dos peixes de águas costeiras de Ponta Negra, Rio Grande do Norte, p.157-165. In: N.T. Chellappa; S. Chellappa \& J.Z.O. Passavante (Eds). Ecologia aquática tropical. Natal, Ed. Servgraf, 165p. Chellappa, S.; F.A. Huntingford; R.H.C. Strang \& R.Y. Thomson. 1995. Condition fator and hepatosomatic index as estimates of energy status in male three-spined stickleback. Journal of Fish Biology, London, 47: 775-787.

Eiras, J.C.; R.M. Takemoto \& G.C. Pavanelli. 2000. Métodos de estudo e técnicas laboratoriais em parasitologia de peixes. Maringá, Editora Universidade Estadual de Maringá, 171p.

Fonteles-Filho, A.A. 1988. Sinopse de informações sobre a Cavala, Scomberomorus cavalla (Cuvier) e a serra, Scomberomorus brasiliensis Collette, Russo \& Zavala-Camin (Pisces: Scombridae), no Estado do Ceará, Brasil. Arquivos de Ciências do Mar, Fortaleza, 27: 21-48.

Huntingford, F.A.; S. Chellappa; A.C. Taylor \& R.H.C. Strang. 2001. Energy reserves and reproductive investment in male three-spined sticklebacks, Gasteroosteus aculeatus. Ecology of freshwater fish, Copenhagen, 10: 111-117.

Iвама. 2003. Boletim Estatístico da Pesca Marítima e Estuarina do Nordeste do Brasil - 2002. Tamandaré, Ministério do Meio Ambiente, IBAMA, CEPENE, 209p.

Luque, J.L.; D. Mouillot \& R. Poulin. 2004. Parasite biodiversity and its determinants in coastal marine teleost fishes of Brazil. Parasitology, Cambridge, 128: 671-682.

Moreira, P.S. 1972. Species of marine isopoda (Crustácea, Peracarida) from southem Brazil. Boletim do Instituto Oceanográfico, São Paulo, 21 (1): 163-179.

Moreira, P.S. 1977. Occurrence and ecological notes on Rocinela signata (Isopoda, Flabellifera) off Brazil. Boletim do Instituto Oceanográfico, São Paulo, 26 (2): 293-301.

Oliva, M.E. \& J.L. Luque. 1998. Metazoan parasites infracommunities in five sciaenids from the Central Peruvian Coast. Memórias do Instituto Oswaldo Cruz, Rio de Janeiro, 93 (2): 175-180.

Pavanelli, G.C.; J.C. Eiras \& R.M. Tакеmoto. 1999. Doenças de peixes, profilaxia, diagnóstico e tratamento. Maringá, Editora Universidade Estadual de Maringá, 264p.

Thatcher, V.E. 2000. The isopod parasites of South American fishes, p.193-226. In: G. Salgado-Maldonado; A.N.G. Aldrett \& V.M. Vidal-Martinez (Eds). Metazoan parasites in the Neotropics: a systematic and ecological perspective. Mexico, Universidad Nacional Autónoma de México Press, 310p.

Thatcher, V.E.; J.M. SouZA-Conceição \& G.F. Jost. 2003. Lironeca desterroensis sp. nov. (Isopoda, Cymothoidae) from the gills of a marine fish, Cetengraulis edentulous Cuvier, of Santa Catarina Island, Brazil. Revista Brasileira de Zoologia, Curitiba, 20 (2): 251-255.

VAZZOLER, A.E.A.M. 1996. Biologia de reprodução de peixes teleósteos: teoria e prática. Maringá, Editora Universidade Estadual de Maringá,169p.

Zavala-Camin, L.A. 1983. Caracterização das espécies brasileiras da família Scombridae (Osteichthyes - Perciformes). Boletim do Instituto de Pesca, São Paulo, 10 (1): 73-93.

Recebido em 06.V.2005; aceito em 14.XI.2005. 